\title{
Asymptotic discord and entanglement of nonresonant harmonic oscillators under weak and strong dissipation
}

\author{
Luis A. Correa, ${ }^{1,2}$ Antonio A. Valido, ${ }^{1,3}$ and Daniel Alonso ${ }^{1,2, *}$ \\ ${ }^{1}$ Instituto Universitario de Estudios Avanzados (IUdEA), Universidad de La Laguna, Tenerife, Canary Islands 38203, Spain \\ ${ }^{2}$ Departamento Física Fundamental, Experimental, Electrónica y Sistemas, Universidad de La Laguna, Tenerife, Canary Islands 38203, Spain \\ ${ }^{3}$ Departamento Física Fundamental II, Universidad de La Laguna, Tenerife, Canary Islands 38203, Spain
}

(Received 29 July 2011; revised manuscript received 20 March 2012; published 20 July 2012)

\begin{abstract}
In this work, we calculate the exact asymptotic quantum correlations between two interacting nonresonant harmonic oscillators in a common Ohmic bath. We derive analytical formulas for the covariances, fully describing any Gaussian stationary state of the system, and use them to study discord and entanglement in the strong- and weak-dissipation regimes. We discuss the rich structure of the discord of the stationary separable states arising in the strong-dissipation regime. Also under strong dissipation, when the modes are not mechanically coupled, these may entangle only through their interaction with the common environment. Interestingly enough, this stationary entanglement assisted by dissipation is only present within a finite band of frequencies and increases with the dissipation rate. Robust entanglement between detuned oscillators is also observed at low temperature.
\end{abstract}

DOI: 10.1103/PhysRevA.86.012110

PACS number(s): 03.65.Yz, 03.65.Ta, 03.67.Mn, 42.50.Lc

\section{INTRODUCTION}

Over the past decades, nonclassical correlations such as discord and entanglement have been widely acknowledged to play a central role in quantum mechanics. In particular, quantum correlations are a valuable resource in quantum communication and information processing with continuous variables (CVs) [1]. Therefore, the investigation of the conditions under which bipartite states indefinitely preserve their nonclassical nature in the presence of noise and dissipation is not only of fundamental importance but also of practical interest. The robustness of entanglement against decoherence in CV systems has been the object of study in a number of recent publications [2-12].

Gaussian two-mode states occupy a privileged position among all entangled CV preparations, since they combine easy experimental realization with simple mathematical description in terms of their second-order moments, which may be experimentally determined via homodyne detection [13]. Gaussian states also find physical realization in mechanical oscillators, such as trapped ions coupled by their electrostatic interaction [14]. A simple but rather general model describing those systems consists of two (detuned) linearly interacting harmonic oscillators in contact with a thermal bath.

Needless to say, entanglement is central to quantum information theory. Particular attention has been placed on CV quantum information, since the preparation, manipulation, and measurement of CV entangled states is relatively simple from the practical point of view [1]. In the following, we use the logarithmic negativity [15] as an entanglement measure.

The notion of quantum discord [16-18] was introduced to capture the quantumness of correlations. Since the majority of quantum states, including most of the separable ones, have nonzero discord [19], it is said to be more general than entanglement as an indicator of nonclassicality. It has recently attracted attention in the field of quantum information now that

\footnotetext{
*dalonso@ull.es
}

it might provide exponential speedup in computational models involving mixed states with vanishingly small entanglement [20], and it has received operational interpretations as a resource for several quantum communication tasks [21]. Unlike entanglement, it does not vanish suddenly in bipartite open quantum systems [22]. It was also found that zero discord between system and environment is a necessary and sufficient condition for completely positive reduced dynamics [23].

The computation of discord is in general very demanding, even for small finite-dimensional systems [24], and some alternative measures have been proposed to overcome this difficulty [25-27]. These have been also extended to the realm of CV systems [28-30]. In this work we are concerned with Gaussian quantum discord [28], which accounts for the nonclassicality as revealed by local Gaussian measurements on one of the modes of a bipartite Gaussian state and for which there are simple analytical expressions.

The stationary entanglement of two resonant modes in a common environment was formally studied in Ref. [3] using the exact Hu-Paz-Zhang (HPZ) master equation. Approximate analytic expressions for the asymptotic entanglement were derived in the zero- and high-temperature limits, as well as in the weak-dissipation case. Other recent works such as Refs. [8,9] addressed the dynamics of quantum correlations between oscillators in common and independent environments, assuming weak dissipation and equal oscillator frequencies. It must be noted that the validity of the results (both analytical and numerical) obtained with this master equation approach has been recently questioned in Ref. [31] whenever nonlocal dissipation effects are important, due to an overlooked mathematical subtlety.

The issue of nonresonant modes was analyzed in Refs. [3,4] with perturbative master equations: For a common environment, it was noted in Ref. [4] that the robustness against sudden death of entanglement critically depended on the frequency matching between the oscillators at high temperatures. Nonetheless, as argued in Ref. [3], nonzero asymptotic entanglement may be achieved out of resonance at sufficiently low temperatures. Much less is known about Gaussian quantum 
discord in this system: Quantum correlations may arise from the interaction with the common environment [9] even if the dissipation is weak. This feature is not present for strictly Markovian dissipation [11].

In this work, we look into the less-explored case of nonresonant oscillators under arbitrarily strong dissipation. We derive exact analytical formulas for the second-order moments of the Gaussian stationary states of two mechanically coupled detuned modes, in contact with a common Ohmic equilibrium environment. Since we proceed by solving the quantum Langevin equations (QLEs) without performing any simplifying assumptions [5,6], our results are exact and therefore apply to the case of strongly non-Markovian systemenvironment interaction just as well as to the case of weak dissipation.

Thus provided with the stationary states of the system, we exactly quantify the asymptotic entanglement and Gaussian quantum discord between the two modes and analyze their dependence on the relevant model parameters. The quantum discord of the stationary separable states arising from strong dissipation displays a rich structure that we report and discuss.

In the absence of mechanical coupling between the modes, their interaction with a common environment may produce entangled asymptotic states $[3,5,12]$. We find that this environmentally assisted entanglement [2,32] vanishes outside a finite range of oscillator frequencies and may be enhanced by increasing the dissipation strength. We also emphasize below the fact that stationary entanglement may actually be found between detuned oscillators at finite temperatures.

The article is structured as follows: The system's Hamiltonian is presented and discussed in the beginning of Sec. II. By choosing a linear system-environment coupling term while keeping the environment general, in Sec. II A we formally obtain the covariances, fully accounting for the stationary Gaussian state of the system, solving the QLEs exactly in Fourier space. In Sec. II B we pick an Ohmic spectral density for the environment to derive explicit analytical formulas for the exact asymptotic covariances. We then briefly consider the weak coupling and low-temperature limit in Sec. IIC. The calculation of the entanglement and Gaussian quantum discord is discussed in Sec. III before we present our results in Sec. IV: The structure of entanglement and discord in the weak-dissipation regime is first analyzed in Sec. IV A, whereas the band structure of entanglement in the absence of mechanical interaction between the modes, as well as the properties of discord of the stationary states with intermediate mechanical couplings under strong dissipation, is discussed in Sec. IV B. Finally, in Sec. V we summarize and conclude.

\section{THE MODEL AND ITS EXACT SOLUTION}

Our system consists of two harmonic oscillators interacting through a term bilinear in the coordinates:

$$
H_{S}=\sum_{i=1}^{2} \frac{p_{i}^{2}}{2 m_{i}}+\frac{1}{2} m_{i} \omega_{i}^{2} x_{i}^{2}+\frac{k}{2}\left(x_{1}-x_{2}\right)^{2} .
$$

The interaction with a thermal environment can be modeled by coupling them to a common bosonic heat bath, which yields

$$
\begin{aligned}
H= & H_{S}+\sum_{\mu}\left(\frac{p_{\mu}^{2}}{2 m_{\mu}}+\frac{1}{2} m_{\mu} \omega_{\mu}^{2} x_{\mu}^{2}\right)+\mathcal{F}\left(x_{1}, x_{2}\right) \sum_{\mu} g_{\mu} x_{\mu} \\
& +\frac{\Omega^{2}}{2} \mathcal{F}\left(x_{1}, x_{2}\right)^{2}
\end{aligned}
$$

where $\Omega^{2}$ is given by $2 \int_{0}^{\infty} d \omega J(\omega) / \omega$, and $J(\omega)$ is the bath spectral density $J(\omega) \equiv \sum_{\mu} g_{\mu} / 2 m_{\mu} \omega_{\mu}$. The last term is introduced to compensate for the renormalization appearing as a result of the system-bath coupling [33]. We use units in which $m_{i}=\hbar=k_{B}=1$.

As noted in Refs. [3,8] among others, under suitable conditions, an initially separable state may become entangled in this model, even if $k=0$. However, this ability of the common environment to create nonclassical correlations between the two parties exponentially decays by increasing the physical distance that separates them [5], so distant dissipative oscillators would be better modeled by considering independent identical environments.

In the following, all averages are taken with respect to a separable initial state in the system's and bath's degrees of freedom $\rho(0) \otimes \rho_{T}$, where $\rho(0)$ is a Gaussian state of the two oscillators and $\rho_{T}$ is a thermal equilibrium state of the environment at temperature $T$.

\section{A. Formal solution of the QLEs}

By choosing $\mathcal{F}\left(x_{1}, x_{2}\right)=x_{1}+x_{2}$ in $H$ one may derive the following quantum Langevin equations [33]:

$$
\begin{aligned}
\ddot{x}_{i} & +\left(\omega_{i}^{2}+\Omega^{2}\right) x_{i}+\sum_{j=1}^{2}\left[k\left(x_{i}-x_{j}\right)+\Omega^{2} x_{j}\right] \tilde{\delta}_{i j} \\
& =F(t)+\sum_{i=1}^{2} \int_{-\infty}^{t} d s \chi(t-s) x_{i}(s),
\end{aligned}
$$

where $\tilde{\delta}_{i j} \equiv 1-\delta_{i j}$. The quantum force $F(t)$ acting on both oscillators and the dissipative kernel $\chi(t)$ are connected by the Kubo relation $\chi(t)=i \theta(t)\langle[F(t), F(0)]\rangle$, where $\theta(t)$ stands for the Heaviside step function. Note that the initial conditions have been taken at $t \rightarrow-\infty$ so that any time $t$ already corresponds to the stationary regime.

For this choice of $\mathcal{F}\left(x_{1}, x_{2}\right)$, the overall system Hamiltonian $H$ is quadratic in positions and momenta, so for $\omega_{1} \neq \omega_{2}$ the asymptotic state [which is independent of $\rho(0)$ ] is guaranteed to be Gaussian.

In the case of resonant oscillators, the normal-mode decomposition leads to an effective decoupling of the relative degree of freedom from the bath, and thus the stationary state would actually depend on the initial condition [3]. In the following we consider detuned oscillators.

Following the approach of Ref. [6], we can solve Eq. (1) by Fourier transformation, which we denote by $\hat{f} \equiv \int_{-\infty}^{\infty} d t e^{i \omega t} f(t)$. Due to the linearity of the problem, this formally yields $\hat{x}_{i}(\omega)=\sum_{j} \alpha_{i j}(\omega) \hat{F}(\omega)$, where the generalized susceptibilities $\alpha_{i j}(\omega)$ are elements of the inverse of the 
matrix

$\alpha^{-1} \equiv\left(\begin{array}{lc}\omega_{1}^{2}+\Omega^{2}-\omega^{2}+k-\hat{\chi} & -k+\Omega^{2}-\hat{\chi} \\ -k+\Omega^{2}-\hat{\chi} & \omega_{2}^{2}+\Omega^{2}-\omega^{2}+k-\hat{\chi}\end{array}\right)$.

The central object of our study is the asymptotic covariance matrix $\Gamma$, defined as $\Gamma_{i j} \equiv\left\langle R_{i} R_{j}\right\rangle-\left[R_{i}, R_{j}\right] / 2$, with $\mathbf{R} \equiv\left\{x_{1}, p_{1}, x_{2}, p_{2}\right\}$ which, up to local displacements, fully characterizes any Gaussian state of our bipartite system [34].

Provided with the susceptibility matrix $\alpha(\omega)$, we may compute the power spectrum $\left\langle x_{i} x_{j}\right\rangle_{\omega} \equiv \int_{-\infty}^{\infty} d t e^{i \omega\left(t-t^{\prime}\right)}\left\langle x_{i}(t-\right.$ $\left.\left.t^{\prime}\right) x_{j}(0)\right\rangle$ of the stationary two-time correlation $\left\langle x_{i}(t-\right.$ $\left.\left.t^{\prime}\right) x_{j}(0)\right\rangle=\left\langle x_{i}(t) x_{j}\left(t^{\prime}\right)\right\rangle$ in terms of the power spectrum of the environmental force:

$$
\left\langle x_{i} x_{j}\right\rangle_{\omega}=\sum_{k, l} \alpha_{i k}(\omega) \alpha_{j l}(-\omega)\langle F F\rangle_{\omega} .
$$

The rest of the power spectra $\left\langle R_{i} R_{j}\right\rangle_{\omega}$ can be readily obtained from Eq. (2) since $\left\langle p_{i} x_{j}\right\rangle_{\omega}=-i \omega\left\langle x_{i} x_{j}\right\rangle_{\omega}$ and $\left\langle p_{i} p_{j}\right\rangle_{\omega}=\omega^{2}\left\langle x_{i} x_{j}\right\rangle_{\omega}$. The full asymptotic covariance matrix then reads

$$
\Gamma_{i j}(t, t)=\int_{-\infty}^{\infty} \frac{\omega}{2 \pi}\left\langle R_{i} R_{j}\right\rangle_{\omega}-\frac{1}{2}\left[R_{i}, R_{j}\right]
$$

\section{B. Analytical covariances for Ohmic dissipation}

To proceed further in deriving analytical expressions, we choose an Ohmic spectral density with Lorentz-Drude cutoff $J(\omega)=2 \gamma \omega / \pi\left(1+\omega^{2} / \omega_{c}^{2}\right)$, which leads to a dissipative kernel $\hat{\chi}(\omega)=2 \gamma \omega_{c}^{2} /\left(\omega_{c}-i \omega\right)$, and a bath correlation function $\langle F F\rangle_{\omega}=\pi J(\omega)[1+\operatorname{coth}(\omega / 2 T)]$, where $\omega_{c}$ is the cutoff frequency and $\gamma$ the dissipation rate. The renormalization constant appearing in Eq. (1) is $\Omega^{2}=2 \gamma \omega_{c}$ for this choice of $J(\omega)$. We start from Eq. (2) to get

$$
\left\langle x_{i}(t) x_{j}(t)\right\rangle=-\int_{-\infty}^{\infty} \frac{d \omega}{2 \pi} \frac{g_{i j}(\omega) \operatorname{coth}\left(\frac{\omega}{2 T}\right)}{h(\omega) h(-\omega)},
$$

where $g_{i j}(\omega)$ and $h(\omega)$ are the fifth-order polynomials

$$
\begin{aligned}
g_{i j}(\omega) & \equiv \frac{\Omega^{2} \omega_{c} \omega\left(2 k+\omega_{1}^{2}-\omega^{2}\right)^{2}\left(2 k+\omega_{2}^{2}-\omega^{2}\right)^{2}}{\left(2 k+\omega_{i}^{2}-\omega^{2}\right)\left(2 k+\omega_{j}^{2}-\omega^{2}\right)}, \\
h(\omega) \equiv & k\left[2 i \omega^{2} \omega_{c}-i\left(\omega_{1}^{2}+\omega_{2}^{2}\right) \omega_{c}-2 \omega^{3}\right. \\
& \left.+\omega\left(\omega_{1}^{2}+\omega_{2}^{2}+4 \Omega^{2}\right)\right]+\omega\left(\omega_{1}^{2}+\omega_{2}^{2}-2 \omega^{2}\right) \Omega^{2} \\
& +\left(\omega^{2}-\omega_{1}^{2}\right)^{2}\left(\omega^{2}-\omega_{2}^{2}\right)^{2}(\omega-i) .
\end{aligned}
$$

The five roots $z_{i}$ of $h(\omega)$ lay in the upper half plane of complex frequencies, as can be checked, and satisfy the Vieta relations:

$$
\begin{aligned}
\sum_{i} z_{i} & =i \omega_{c}, \quad \sum_{i<j} z_{i} z_{j}=-2\left(\Omega^{2}+k\right)-\omega_{1}^{2}-\omega_{2}^{2}, \\
\sum_{i<j<k} z_{i} z_{j} z_{k} & =-i \omega_{c}\left(2 k+\omega_{1}^{2}+\omega_{2}^{2}\right), \\
\sum_{i<j<k<l} z_{i} z_{j} z_{k} z_{l} & =\left(k+\Omega^{2}\right)\left(\omega_{1}^{2}+\omega_{2}^{2}\right)+4 \Omega^{2} k+\omega_{1}^{2} \omega_{2}^{2}, \\
\prod_{i} z_{i} & =i \omega_{c}\left[k\left(\omega_{1}^{2}+\omega_{2}^{2}\right)+\omega_{1}^{2} \omega_{2}^{2}\right] .
\end{aligned}
$$

We now replace coth $(\omega / 2 T)$ in Eq. (3) by the expansion

$$
\operatorname{coth}\left(\frac{\omega}{2 T}\right)=2 T \sum_{n=-\infty}^{\infty} \frac{\omega}{\omega^{2}+v_{n}^{2}},
$$

where $v_{n} \equiv 2 \pi n T$ are the Matsubara frequencies [35], and we exchange the summation with the integral sign. The expression is thus transformed into

$$
\begin{aligned}
\left\langle x_{i}(t) x_{j}(t)\right\rangle= & -T \int_{-\infty}^{\infty} \frac{d \omega}{\pi} \frac{g_{i j}(\omega) / \omega}{h(\omega) h(-\omega)} \\
& +2 T \sum_{n=1}^{\infty} \int_{-\infty}^{\infty} \frac{d \omega}{\pi} \frac{\omega g_{i j}(\omega)}{\tilde{h}(\omega) \tilde{h}(-\omega)},
\end{aligned}
$$

where $\tilde{h}(\omega) \equiv\left(\omega-i v_{n}\right) h(\omega)$.

We may now use the formula given in Sec. 3.1.1 of Ref. [36] to explicitly solve the integrals, which yields

$$
\begin{aligned}
\left\langle x_{i}(t) x_{j}(t)\right\rangle= & \frac{T\left(k \omega_{i}^{2}+\delta_{i j} \omega_{1}^{2} \omega_{2}^{2}\right)}{k\left(\omega_{1}^{2}+\omega_{2}^{2}\right) \omega_{i}^{2}+\omega_{1}^{2} \omega_{2}^{2} \omega_{i}^{2}} \\
& -2 i T \sum_{n=1}^{\infty}\left[\frac{k\left(\omega_{c}+v_{n}\right)-\Omega^{2} v_{n}}{h\left(-i v_{n}\right)}\right. \\
& \left.+\delta_{i j} \frac{2 \Omega^{2} v_{n}+\left(v_{n}^{2}+\omega_{1}^{2} \omega_{2}^{2} / \omega_{i}^{2}\right)\left(\omega_{c}+v_{n}\right)}{h\left(-i v_{n}\right)}\right] .
\end{aligned}
$$

The sum above can be further simplified by using the identity [33]

$$
\sum_{i=1}^{N} a_{i} \sum_{n=1}^{\infty} \frac{1}{n+z_{i}}=-\sum_{i=1}^{N} a_{i} \psi\left(1+z_{i}\right)
$$

where $\psi(z) \equiv d \ln \Gamma(z) / d z$ stands for the logarithmic derivative of Euler's $\Gamma$ function. The formula holds if $\sum_{i}^{N} a_{i}=0$, which turns out to be the case. The following compact formula for the stationary position-position correlations is thus finally obtained:

$$
\begin{aligned}
\left\langle x_{i}(t) x_{j}(t)\right\rangle= & \frac{T\left(k \omega_{i}^{2}+\delta_{i j} \omega_{1}^{2} \omega_{2}^{2}\right)}{k\left(\omega_{1}^{2}+\omega_{2}^{2}\right) \omega_{i}^{2}+\omega_{1}^{2} \omega_{2}^{2} \omega_{i}^{2}} \\
& -\sum_{k=1}^{5} a_{k}^{(i, j)} \psi\left(1-\frac{i z_{k}}{v_{1}}\right) .
\end{aligned}
$$

Note that this equals the classical mean value $\left\langle x_{i} x_{j}\right\rangle_{T \rightarrow \infty}$, minus a quantum correction term.

The coefficients $a_{k}^{(i, j)}$ are explicitly given by

$$
\begin{aligned}
a_{k}^{(i, j)}= & \frac{k \omega_{c}-i z_{k}\left(\Omega^{2}-k\right)}{\pi \prod_{l \neq k}\left(z_{k}-z_{l}\right)} \\
& +\delta_{i j} \frac{\left(\omega_{c}+i z_{k}\right) \omega_{1}^{2} \omega_{2}^{2} / \omega_{i}^{2}+2 i \Omega^{2} z_{k}-\omega_{c} z_{k}^{2}-i z_{k}^{3}}{\pi \prod_{l \neq k}\left(z_{k}-z_{l}\right)} .
\end{aligned}
$$

To complete the analytical characterization of the exact stationary covariances, we now turn our attention to the momentum-momentum correlations $\left\langle p_{i} p_{j}\right\rangle_{\omega}$, since the rest of the elements of the covariance matrix (namely $\operatorname{Re}\left\langle x_{i}(t) p_{j}(t)\right\rangle$ ), are all zero as may be seen by analogous calculations:

$$
\left\langle p_{i}(t) p_{j}(t)\right\rangle=-\int_{-\infty}^{\infty} \frac{d \omega}{2 \pi} \frac{\omega^{2} g_{i j}(\omega) \operatorname{coth}\left(\frac{\omega}{2 T}\right)}{h(\omega) h(-\omega)} .
$$


This may be evaluated in exactly the same way as we did for the position-position correlations, to yield

$$
\begin{aligned}
\left\langle p_{i}(t) p_{j}(t)\right\rangle= & T \delta_{i j}-2 i T \sum_{n=1}^{\infty} \frac{\Omega^{2} v_{n}^{3}-k\left(\omega_{c}+v_{n}\right) v_{n}^{2}}{h\left(-i v_{n}\right)}-2 i T k \delta_{i j} \sum_{n=1}^{\infty} \frac{\left(\omega_{1}^{2}+\omega_{2}^{2}\right)\left(\omega_{c}+v_{n}\right)+v_{n}\left[4 \Omega^{2}+2 v_{n}\left(\omega_{c}+v_{n}\right)\right]}{h\left(-i v_{n}\right)} \\
& -2 i T \delta_{i j} \sum_{n=1}^{\infty} \frac{\Omega^{2} v_{n} \omega_{1}^{2} \omega_{2}^{2} / \omega_{i}^{2}+\omega_{1}^{2} \omega_{2}^{2}\left(\omega_{c}+v_{n}\right)+\omega_{i}^{2} v_{n}\left[\Omega^{2}+v_{n}\left(\omega_{c}+v_{n}\right)\right]}{h\left(-i v_{n}\right)} .
\end{aligned}
$$
with

After explicitly performing all the summations, we are left

$$
\left\langle p_{i}(t) p_{j}(t)\right\rangle=T \delta_{i j}-\sum_{k=1}^{5} b_{k}^{(i, j)} \psi\left(1-i \frac{z_{k}}{v_{1}}\right) .
$$

Note again that $T \delta_{i j}$ equals the classical average $\left\langle p_{i} p_{j}\right\rangle_{T \rightarrow \infty}$. As it is straightforward to check, the coefficients $b_{k}^{(i, j)}$ are given by

$$
\begin{aligned}
b_{k}^{(i, j)}= & \frac{\left[k \omega_{c}+i\left(k-\Omega^{2}\right) z_{k}\right] z_{k}^{2}}{\pi \prod_{l \neq k}\left(z_{k}-z_{l}\right)} \\
& +\delta_{i j} \frac{\left[\omega_{c} k+i\left(\Omega^{2}+k\right) z_{k}\right]\left(\omega_{1}^{2}+\omega_{2}^{2}\right)}{\pi \prod_{l \neq k}\left(z_{k}-z_{l}\right)} \\
& +\delta_{i j} \frac{\left(\omega_{c}+i z_{k}\right) \omega_{1}^{2} \omega_{2}^{2}+4 i k \Omega^{2} z_{k}}{\pi \prod_{l \neq k}\left(z_{k}-z_{l}\right)} \\
& -\delta_{i j} \frac{\left(2 k+\omega_{i}^{2}\right)\left(\omega_{c} z_{k}^{2}+i z_{k}^{3}\right)}{\pi \prod_{l \neq k}\left(z_{k}-z_{l}\right)} .
\end{aligned}
$$

\section{Weak dissipation and low-temperature limit}

Now that we have the exact analytical stationary covariances, we can gain some insight into the physics of the problem by setting $k=0$ and taking the limit $\gamma / \omega_{c} \ll 1$. To first order in this small parameter, the five roots $z_{k}$ of $h(\omega)$ become

$$
\begin{aligned}
z_{1,2,3,4} & = \pm \omega_{i} \pm \frac{\gamma \omega_{c}}{\left(\omega_{i} \mp i \omega_{c}\right)}, \\
z_{5} & =i \omega_{c}-\frac{i 2 \gamma \omega_{c}^{2}\left(\omega_{1}^{2}+\omega_{2}^{2}+2 \omega_{c}^{2}\right)}{\prod_{i}\left(\omega_{i}^{2}+\omega_{c}^{2}\right)} .
\end{aligned}
$$

Additionally, in the very-low-temperature regime (i.e., $\left.T \ll \omega_{i}\right)$ the asymptotic expansion $\psi(1+z) \simeq \ln z+(2 z)^{-1}$ may be justified. Inserting all this into the formulas of the covariances derived above, and retaining terms only to first order in $T / \omega_{c}, \omega_{i} / \omega_{c}$, and $\gamma / \omega_{c}$, one gets the temperatureindependent expressions

$$
\begin{aligned}
\left\langle x_{i}^{2}\right\rangle & \simeq \frac{1}{2 \omega_{i}}-\frac{\gamma}{2 \pi}\left[\frac{2 \omega_{c}-\pi \omega_{i}}{\omega_{i}^{2} \omega_{c}}+\frac{4}{\omega_{c}^{2}}\left(\ln \frac{\omega_{c}}{\omega_{i}}\right)-\frac{1}{2}\right], \\
\left\langle p_{i}^{2}\right\rangle & \simeq \frac{1}{2} \omega_{i}+\frac{\gamma}{2 \pi}\left[3 \pi \frac{\omega_{i}}{\omega_{c}}-2+4 \ln \frac{\omega_{c}}{\omega_{i}}\right], \\
\left\langle x_{1} x_{2}\right\rangle & \simeq \frac{\gamma}{\pi\left(\omega_{1}^{2}-\omega_{2}^{2}\right)} 2 \ln \frac{\omega_{2}}{\omega_{1}}, \\
\left\langle p_{1} p_{2}\right\rangle & \simeq \frac{\gamma \ln 16}{2 \pi}+\frac{\gamma\left(\omega_{1}^{2} \ln \frac{\omega_{c}^{2}}{4 \omega_{1}^{2}}-\omega_{2}^{2} \ln \frac{\omega_{c}^{2}}{4 \omega_{2}^{2}}\right)}{\pi\left(\omega_{1}^{2}-\omega_{2}^{2}\right)} .
\end{aligned}
$$

By setting $\gamma=0$, the variances $\left\langle x_{i}^{2}\right\rangle$ and $\left\langle p_{i}^{2}\right\rangle$ become those of the ground state of the corresponding oscillators without dissipation, and the asymptotic (separable) state has a diagonal covariance matrix. Taking into account that $\omega_{c} / \omega_{i} \gg 1$, one sees that increasing $\gamma$ leads to a reduction of $\left\langle x_{i}^{2}\right\rangle$ while $\left\langle p_{i}^{2}\right\rangle$ gets larger, as expected. The terms proportional to $\log \omega_{c} / \omega_{i}$ in $\left\langle p_{i} p_{j}\right\rangle$ also occur in the same region of parameters for a single damped oscillator [33].

\section{QUANTUM CORRELATIONS}

The asymptotic entanglement and discord between the modes may be readily estimated from the stationary covariance matrix $\Gamma$. We consider the logarithmic negativity $\left(E_{\mathcal{N}}\right)[15]$ as a measure of entanglement. It is based on the positivity of the partial transpose (PPT) [37], which turns out to be a necessary and sufficient separability criterion for bipartite Gaussian states [38], and is computed from the lowest symplectic eigenvalue $\tilde{v}_{-}$of the partially transposed covariance matrix [15] as

$$
E_{\mathcal{N}}=\max \left\{0,-\ln 2 \tilde{v}_{-}\right\}
$$

When it comes to discord $(\mathcal{D})[16,17]$, it is defined as the difference between two classically equivalent definitions of quantum mutual information:

$$
\mathcal{D}^{\leftarrow}\left(\rho_{12}\right)=I\left(\rho_{12}\right)-\mathcal{I}^{\leftarrow}\left(\rho_{12}\right),
$$

where the total correlations are given by $I\left(\rho_{12}\right)=S\left(\rho_{12}\right)+$ $S\left(\rho_{12}\right)-S\left(\rho_{12}\right)$ and the classical correlations $[17] \mathcal{I}^{\leftarrow}\left(\rho_{12}\right)=$ $\max _{\left\{\Pi_{i}^{(2)}\right\}}\left\{S\left(\rho_{12}\right)-\sum_{i} p_{i} S\left(\rho_{1}^{(i)}\right)\right\}$. Here the optimization runs over all positive operator-valued measurements (POVMs) $\left\{\Pi_{i}^{(2)}\right\}$ of subsystem $2 ; p_{i}=\operatorname{tr}_{12}\left\{\rho_{12} \Pi_{i}^{(2)}\right\}$ is the probability associated with the $i$ th measurement outcome and $\rho_{1}^{(i)}=$ $\operatorname{tr}_{2}\left\{\rho_{12} \Pi_{i}^{(2)}\right\} / p_{i}$ is the corresponding postmeasurement reduced state of subsystem 1 . Here $S(\cdots)$ stands for von Neumann entropy. $\mathcal{D}^{\leftarrow}$ is in general nonsymmetric under exchange of the parties 1 and $2\left(\mathcal{D}^{\leftarrow} \neq \mathcal{D}^{\rightarrow}\right)$, and in the following we are only concerned with $\mathcal{D}^{\leftarrow}$.

To calculate Gaussian quantum discord $\left(\mathcal{D}_{\mathcal{G}}\right)$, one restricts the optimization to Gaussian POVMs $\left\{\Pi_{\mathcal{G}, i}^{(2)}\right\}$. An explicit formula for $\mathcal{D}_{\mathcal{G}}$ exists, in terms of the four local symplectic invariants of any input Gaussian state $\rho_{12}^{\mathcal{G}}$ [28].

\section{RESULTS AND DISCUSSION}

\section{A. Weak dissipation}

We look first into the weak-dissipation regime, where the relaxation time scale $\tau_{R}=\gamma^{-1}$ is much larger than the bath correlation time, given by $\tau_{B}=v_{1}^{-1}$ whenever $\omega_{c}>v_{1}$ [35]. We also pick a low temperature $T$ for which nonzero 

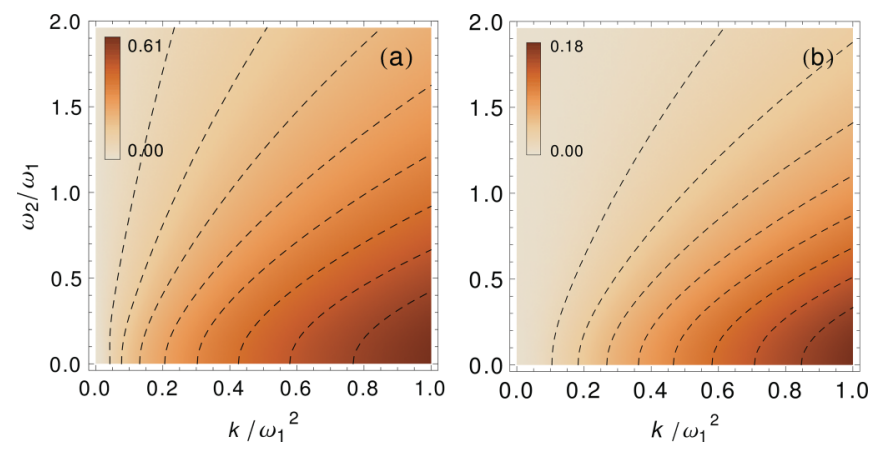

FIG. 1. (Color online) (a) Asymptotic entanglement and (b) discord in the weak-coupling regime as functions of $k$ and $\omega_{2}$. We have chosen $\omega_{1}=10, \gamma=0.01, \omega_{c}=500$, and $T=0.5$. Dashed lines are contours and the color legends are plotted in the upper left corners.

asymptotic entanglement might exist. Results are presented in Fig. 1. In accordance with Ref. [3], we observe $E_{\mathcal{N}} \neq 0$ out of resonance at low temperatures, and overall similar behavior of entanglement and discord.

As can be seen, both measures of quantum correlations increase with $\omega_{2} / k \rightarrow 0$. This may be understood as follows: In the limit $k \gg \omega_{i}$, the oscillators, whose effective frequency $\tilde{\omega}^{2}$ is given by $\omega_{i}^{2}+k+2 \gamma \omega_{c}$, become nearly resonant and, thus, approximately decouple in the variables $\eta_{ \pm} \equiv\left(x_{1} \pm x_{2}\right) / \sqrt{2}$ and $\pi_{ \pm} \equiv\left(p_{1} \pm p_{2}\right) / \sqrt{2}$. As $\omega_{i} / k \rightarrow 0,\left\{\left\langle\eta_{+}^{2}\right\rangle,\left\langle\pi_{-}^{2}\right\rangle\right\} \rightarrow \infty$ while $\left\{\left\langle\pi_{+}^{2}\right\rangle,\left\langle\eta_{-}^{2}\right\rangle\right\} \rightarrow 0[6]$. This corresponds to a nonsymmetric two-mode squeezed thermal state with infinite squeezing $r$, i.e., the ideal Einstein-Podolsky-Rosen (EPR) (maximally entangled) state [39].

\section{B. Strong dissipation}

We achieve this regime by increasing $\gamma$, so that the relaxation time $\tau_{R}$ becomes comparable to the bath correlation time $\tau_{B}$.

Due to the bigger decoherence, entanglement is mostly lost, though its maximum is still attained as $\omega_{2} / k \rightarrow 0$. Interestingly, in the inset of Fig. 2(a), we can observe a small region around $k=0$ where nonclassical correlations are enhanced by the dissipation. This purely non-Markovian effect enters into our equations through the renormalization term $\Omega^{2} x_{1} x_{2}$ in the Hamiltonian $H$, which for sufficiently high dissipation rate results in an effective environment-assisted coupling between the oscillators. Actually, even at $k=0$, there may exist nonvanishing stationary entanglement. The corresponding asymptotic states are thus non-Gibbsian, as could be expected at sufficiently low temperatures [40].

One sees in Fig. 2(b) that for fixed $\omega_{1}, \gamma, T$, and $\omega_{c}$ there exists a band of frequencies $\omega_{2} \in\left[\omega^{\prime}, \omega^{\prime \prime}\right]$ for which the asymptotic state is nonseparable. As the temperature increases, the amount of entanglement gets reduced, while $\omega^{\prime}$ and $\omega^{\prime \prime}$ come closer to each other. On the contrary, by increasing the dissipation rate $\gamma$, the maximum attainable $E_{\mathcal{N}}$ and the bandwidth $\omega^{\prime \prime}-\omega^{\prime}$ get larger. Reducing the frequency $\omega_{1}$ also leads to narrower bandwidth. One may also see that $\omega^{\prime}$ is almost insensitive to changes in $\omega_{c}$, while $\omega^{\prime \prime}$ slightly decreases as $\omega_{c}$ grows.
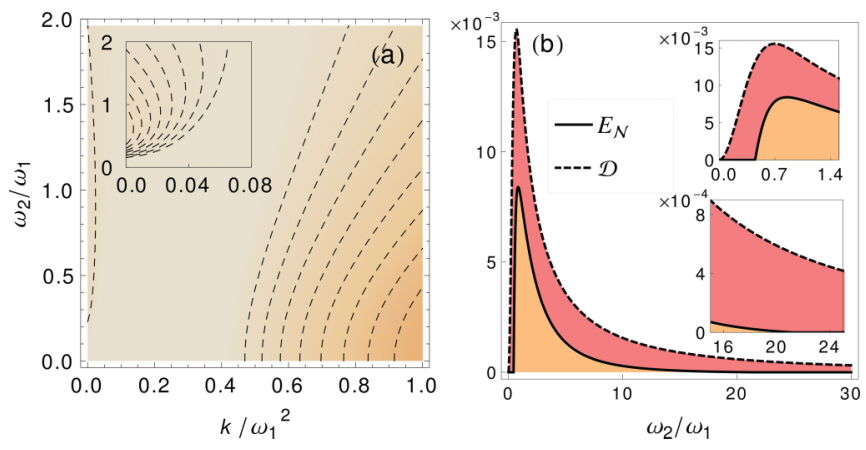

FIG. 2. (Color online) (a) Asymptotic entanglement in the strongdissipation regime as a function of $k$ and $\omega_{2}$. In the inset, we are zooming into the region of low mechanical coupling. (b) Asymptotic entanglement (continuous line) and discord (dashed line) as functions of $\omega_{2}$ alone for $k=0$. For both plots, parameters are the same as in Fig. 1 except for the dissipation $\gamma=0.5$.

The simple model $H_{S}=\sum_{i}\left[p_{i}^{2} / 2+\left(\omega_{i}^{2}+\Omega^{2}\right) x_{i}^{2} / 2\right]+$ $\Omega^{2} x_{1} x_{2}$ proves useful to understand these features. Note that the renormalization $\Omega^{2}=2 \gamma \omega_{c}$ has been introduced to account for the bath-mediated coupling. If $w_{i} / \Omega^{2} \ll 1$, the oscillators are again near resonance and approximately decouple in the coordinates $\left\{\eta_{ \pm}, \pi_{ \pm}\right\}$. Then, $E_{\mathcal{N}} \neq 0$ whenever $\tilde{v}_{-}^{2}=\left\langle\eta_{-}^{2}\right\rangle\left\langle\pi_{+}^{2}\right\rangle<1 / 4[6]$.

It is easy to see that the lowest symplectic eigenvalue of the partially transposed covariance matrix $\tilde{v}_{-}$grows with $T$ and decreases with $\Omega^{2}$ and $\omega_{i}$. Thus, an increase in the temperature must be compensated by an increase in $\omega^{\prime}$ for the inequality to be satisfied. Conversely, an increase in either $\Omega^{2}$ or $\omega_{1}$ allows for a reduction of the critical frequency $\omega^{\prime}$. The existence of an upper bound $\omega^{\prime \prime}$ for this nonseparable region is related to the structure of the bath, so its behavior cannot be inferred from this simple nondissipative model.

We also remark that in our simplified model $\tilde{v}_{-}$decreases as $\left|\Omega^{2}-k\right|$ grows, since the effective coupling between the oscillators is the net result of two competing effects that may eventually cancel each other: the environment-assisted coupling and the direct interaction between the oscillators. This explains qualitatively why $E_{\mathcal{N}}=0$ for intermediate couplings in Fig. 2.

Finally, Fig. 3 contains results on discord, which behaves rather differently in the strong-dissipation regime: Since the entanglement has considerably decreased, it no longer dominates over other quantum correlations and, as could be expected, remains nonzero even when the asymptotic state is separable. It displays a maximum around $k \simeq 20$, marked with the dotted line in Fig. 3, and also vanishes in the limit $\left\{k, \omega_{2}\right\} \rightarrow 0$.

The global purity $\mu$ may be found to be roughly constant for all stationary states, except those with $\left\{k, \omega_{2}\right\} \rightarrow 0$, while $\tilde{v}_{-}$maximizes on the dotted line of Fig. 3, which corresponds to separable states. This suggests that at fixed $\mu$, Gaussian quantum discord increases with $\tilde{v}_{-}$provided that $\tilde{v}_{-}>1 / 2$ (i.e., for separable states), which is consistent with Ref. [28], where this was seen to hold for squeezed thermal states. Additionally, since the partial purity $\mu_{2} \rightarrow 0$ as $\left\{k, \omega_{2}\right\} \rightarrow 0$ the marginal stationary states $\rho_{2} \equiv \operatorname{tr}_{1}\left(\rho_{12}\right)$ become maximally 


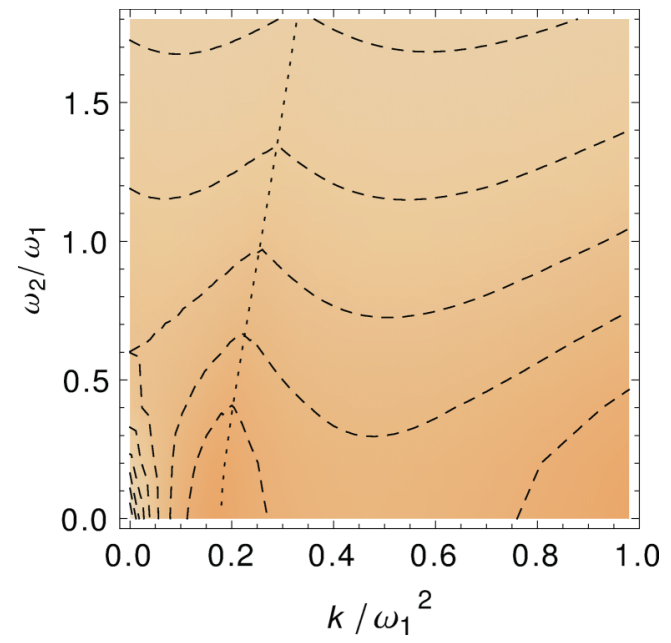

FIG. 3. (Color online) Asymptotic discord in the strongdissipation regime. The dotted line highlights the local maximum of discord for the separable stationary states at intermediate mechanical coupling strengths. All parameters are the same as in Fig. 2.

mixed in this limit, therefore yielding a quantum-classical state [26] with zero discord as revealed by measurements on mode 2 .

At higher temperatures and stronger dissipation rates, the fate of the asymptotic entanglement is to disappear completely, in contrast with the asymptotic quantum discord, which is always nonzero as could be expected [19].

\section{CONCLUSIONS}

We have exactly solved the problem of the asymptotic quantum correlations in a system of two mechanically coupled nonresonant harmonic oscillators in a common bath. In the case of Ohmic dissipation, we provided analytical formulas for the exact covariances, fully characterizing any Gaussian stationary state of the system, and used them to compute the asymptotic entanglement, as measured by the logarithmic negativity, and the Gaussian quantum discord, in both the weak- and strong-dissipation regimes. It is worth stressing that our results do not rely on any simplifying assumptions concerning the different time scales of the problem (e.g., weak system-environment interaction).

We found that robust entanglement between nonresonant oscillators may exist at sufficiently low temperatures. Furthermore, for a finite band of frequencies, it may be created only due to the environment-assisted interaction between the two modes, in the strong-dissipation regime. Also in this regime we have discussed the nontrivial structure of the quantum discord of the stationary separable states, corresponding to intermediate mechanical interaction strengths.

Note added. Recently, another paper appeared [41] where the dynamics of Gaussian quantum discord between resonant oscillators in a common environment was studied within $\mathrm{Hu}$ Paz-Zhang master equation formalism.

\section{ACKNOWLEDGMENTS}

The authors are grateful to José P. Palao for fruitful discussion and to the Spanish MICINN (Grant No. FIS201019998) for funding. L.A.C. and A.A.V. gratefully acknowledge the Canary Islands Government for financial support through the ACIISI fellowships (85\% cofunded by the European Social Fund). D.A. is grateful to E. Sentís Rodríguez for useful comments.
[1] S. L. Braunstein and P. van Loock, Rev. Mod. Phys. 77, 513 (2005).

[2] M. B. Plenio and S. F. Huelga, Phys. Rev. Lett. 88, 197901 (2002).

[3] J. P. Paz and A. J. Roncaglia, Phys. Rev. Lett. 100, 220401 (2008); Phys. Rev. A 79, 032102 (2009).

[4] F. Galve, G. L. Giorgi, and R. Zambrini, Phys. Rev. A 81, 062117 (2010).

[5] T. Zell, F. Queisser, and R. Klesse, Phys. Rev. Lett. 102, 160501 (2009).

[6] M. Ludwig, K. Hammerer, and F. Marquardt, Phys. Rev. A 82, 012333 (2010).

[7] F. Galve, L. A. Pachón, and D. Zueco, Phys. Rev. Lett. 105, 180501 (2010).

[8] K.-L. Liu and H.-S. Goan, Phys. Rev. A 76, 022312 (2007).

[9] R. Vasile, S. Olivares, M. G. A. Paris, and S. Maniscalco, Phys. Rev. A 80, 062324 (2009); R. Vasile, P. Giorda, S. Olivares, M. G. A. Paris, and S. Maniscalco, ibid. 82, 012313 (2010).

[10] J.-H. An and W.-M. Zhang, Phys. Rev. A 76, 042127 (2007).

[11] A. Isar, Open Syst. Inf. Dyn. 18, 175 (2011).

[12] A. Wolf, G. De Chiara, E. Kajari, E. Lutz, and G. Morigi, Europhys. Lett. 95, 60008 (2011).
[13] V. D'Auria, S. Fornaro, A. Porzio, S. Solimeno, S. Olivares, and M. G. A. Paris, Phys. Rev. Lett. 102, 020502 (2009).

[14] K. Brown, C. Ospelkaus, Y. Colombe, A. Wilson, D. Leibfried, and D. Wineland, Nature (London) 471, 196 (2011); M. Harlander, R. Lechner, M. Brownnutt, R. Blatt, and W. Hänsel, ibid. 471, 200 (2011).

[15] G. Vidal and R. F. Werner, Phys. Rev. A 65, 032314 (2002).

[16] W. H. Zurek, Annalen der Physik 9, 855 (2000); H. Ollivier and W. H. Zurek, Phys. Rev. Lett. 88, 017901 (2001).

[17] L. Henderson and V. Vedral, J. Phys. A: Math. Gen. 34, 6899 (2001)

[18] K. Modi, A. Brodutch, H. Cable, T. Paterek, and V. Vedral, arXiv:1112.6238v1.

[19] A. Ferraro, L. Aolita, D. Cavalcanti, F. M. Cucchietti, and A. Acín, Phys. Rev. A 81, 052318 (2010).

[20] A. Datta, A. Shaji, and C. M. Caves, Phys. Rev. Lett. 100, 050502 (2008); B. P. Lanyon, M. Barbieri, M. P. Almeida, and A. G. White, ibid. 101, 200501 (2008).

[21] V. Madhok and A. Datta, Phys. Rev. A 83, 032323 (2011); B. Dakic, Y. O. Lipp, X. Ma, M. Ringbauer, S. Kropatschek, S. Barz, T. Paterek, V. Vedral, A. Zeilinger, C. Brukner, and P. Walther (2012), arXiv:1203.1629v1; M. Gu, H. M. 
Chrzanowski, S. M. Assad, T. Symul, K. Modi, T. C. Ralph, V. Vedral, and P. K. Lam (2012), arXiv:1203.0011v1.

[22] T. Werlang, S. Souza, F. F. Fanchini, and C. J. Villas Boas, Phys. Rev. A 80, 024103 (2009).

[23] A. Shabani and D. A. Lidar, Phys. Rev. Lett. 102, 100402 (2009).

[24] S. Luo, Phys. Rev. A 77, 042303 (2008).

[25] S. Luo, Phys. Rev. A 77, 022301 (2008).

[26] M. Piani, P. Horodecki, and R. Horodecki, Phys. Rev. Lett. 100, 090502 (2008); S. Wu, U. V. Poulsen, and K. Mølmer, Phys. Rev. A 80, 032319 (2009).

[27] B. Dakić, V. Vedral, and C. Brukner, Phys. Rev. Lett. 105, 190502 (2010); S. Luo and S. Fu, Phys. Rev. A 82, 034302 (2010).

[28] G. Adesso and A. Datta, Phys. Rev. Lett. 105, 030501 (2010); P. Giorda and M. G. A. Paris, ibid. 105, 020503 (2010).

[29] L. Mišta, R. Tatham, D. Girolami, N. Korolkova, and G. Adesso, Phys. Rev. A 83, 042325 (2011)

[30] G. Adesso and D. Girolami, Int. J. Quantum. Inf. 9, 1773 (2011).

[31] C. Fleming, A. Roura, and B. Hu, Ann. Phys. 326, 1207 (2011).
[32] C. A. Muschik, E. S. Polzik, and J. I. Cirac, Phys. Rev. A 83, 052312 (2011).

[33] U. Weiss, Quantum Dissipative Systems, Vol. 13 (World Scientific, Singapore, 2008).

[34] A. Ferraro, S. Olivares, and M. Paris, arXiv:quant-ph/0503237v1.

[35] H. Breuer and F. Petruccione, The Theory of Open Quantum Systems (Oxford University Press, New York, 2002).

[36] I. S. Gradshteyn and I. M. Ryzhik, Table of Integrals, Series, and Products, 7th ed. (Academic Press, New York, 2007).

[37] A. Peres, Phys. Rev. Lett. 77, 1413 (1996); M. Horodecki, P. Horodecki, and R. Horodecki, Phys. Lett. A 223, 1 (1996).

[38] R. Simon, Phys. Rev. Lett. 84, 2726 (2000).

[39] A. Einstein, B. Podolsky, and N. Rosen, Phys. Rev. 47, 777 (1935).

[40] T. M. Nieuwenhuizen and A. E. Allahverdyan, Phys. Rev. E 66, 036102 (2002).

[41] J. N. Freitas and J. P. Paz, Phys. Rev. A 85, 032118 (2012). 